\title{
Polyurethane Elastomers as Maxillofacial Prosthetic Materials
}

\author{
A. Jon Goldberg, Robert G. Craig, ${ }^{*}$ and Frank E. Filisko
}

Department of Restorative Dentistry, The University of Connecticut Health Center, Farmington, Connecticut 06032, USA, *Department of Dental Materials, School of Dental Materials, School of Dentistry, The University of Michigan, Ann Arbor, Michigan 48109, USA, and $\dagger$ Department of Materials and Metallurgical Engineering, College of Engineering, The University of Michigan, Ann Arbor, Michigan 48109, USA

A series of polyurethane elastomers based on an aliphatic diisocyanate and a polyether macroglycol was polymerized with various crosslink densities and $O H / N C O$ ratios. Stoichiometries yielding between 8,600 and $12,900 \mathrm{gm} /$ mole/crosslink and an $\mathrm{OH} / \mathrm{NCO}$ ratio of 1.1 resulted in polymers with the low modulus, yet high strength and elongation necessary for maxillofacial applications.

J Dent Res 57 (4) :563-569, April 1978.

There are no completely satisfactory maxillofacial reconstructive materials. The best maxillofacial prosthesis available today, although excellent when originally delivered, deteriorates in 6 to 12 months to the point where it requires replacement. ${ }^{1-4}$ This deterioration is associated with either degradation of mechanical properties or changes in appearance. The deficient properties which most commonly cause mechanical failure are tear resistance and general stiffening of the elastic material as a result of migration and leaching of the plasticizer. Degradation of esthetic qualities can be a result of color changes in the base polymer caused by oxidation and ultraviolet light, color changes in pigments and dyes used to characterize the prosthesis, or adsorption of dirt, grease, or cosmetics onto the surface and subsequent diffusion into the polymer.

Gurrently, the most widely used types of maxillofacial materials include rigid poly(methyl methacrylate), plasticized poly (vinyl chloride) or plasticized vinyl chloride-vinyl acetate copolymers, and silicone rubbers. Sev-

Received for publication September 1, 1977.

Accepted for publication October 25, 1977.

This investigation was supported by USPHS Training Grant DE-00181 from the National Institute of Dental Research, National Institutes of Health, Bethesda, Md 20014.

Portions of this article were presented at the AADR Meeting, New York, 1975. eral other materials have recently been suggested including a latex-dispersed elastomer, ${ }^{5}$ a silphenylene polymer, ${ }^{6}$ and a polycarbonate/ silicone rubber block copolymer. ${ }^{7}$

Polyurethane elastomers have great potential as maxillofacial materials. This hypothesis is based on their inherent environmental stability, high tear resistance, low modulus without the use of plasticizers, and good ultimate strength and elongation. They can accept intrinsic coloring and are amenable to maxillofacial processing techniques. Most importantly, the structure of the polyurethane polymer can be varied to optimize the desired properties and as a result polyurethanes are currently used in a variety of biomaterial applications. ${ }^{8-21}$

Polyurethanes are based on component diisocyanates, macroglycols, chain extenders, and crosslinking agents. In order to develop a satisfactory maxillofacial material the optimum combination of chemistry and morphology must be utilized. Comprehensive literature reviews of polyurethane structure-property relations and chemistry, including discussions of molecular weight, intermolecular forces, chain stiffness, tendency toward crystallization, and intermolecular bonding, can be found elsewhere. ${ }^{22-25}$ One unexpected aspect of crosslinking, however, does warrant brief description. Thermoset urethane elastomers are produced through the introduction of primary chemical crosslinks. In most practical polyurethane systems, the crosslinking is a complex combination of bonds resulting from trifunctional chain extenders, allophanate and biuret links, and physical crosslinks associated with paracrystalline domains. ${ }^{26,27}$ However, if the reactants are combined in stoichiometric ratios, and the reactions are preferentially catalyzed, a known, controlled morphology can be 


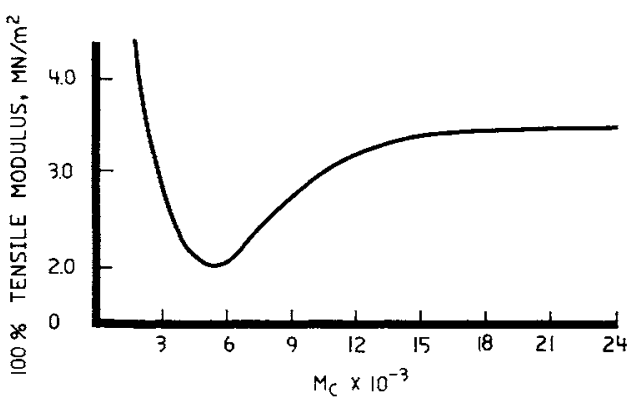

FIG 1.-100\% tensile modulus versus molecular weight per crosslink for a poly(ester urethane) elastomer. (Adapted from Pigott.)

developed. This approach was used by Pigott ${ }^{28}$ to evaluate the effect of crosslink density on the tensile properties of poly (ester urethanes). An interesting minimum occurred in the plot of $100 \%$ modulus versus molecular weight per crosslink, reproduced in Figure 1. This artifact is contrary to the increasing modulus with crosslink density seen with hydrocarbon elastomers, and can be explained on the basis of the secondary bonding present in poly'ester urethanes). As crosslinks are added to the polymer, the initial effect is to inhibit alignment of the polyurethane chains, decreasing the amount of secondary bonding, and hence the modulus and strength. With continued increased crosslink concentration a point is reached (molecular weight per crosslink of approximately 5,000) where the crosslinks themselves start to support a significant amount of the load. Further increases in crosslink density now raise the $100 \%$ modulus and strength.

On the basis of the knowledge of polyurethane elastomers it was hypothesized that a polyether macroglycol in combination with an aliphatic diisocyanate could provide the necessary environmental stability for a maxillofacial material. This polyether-aliphatic molecule is inherently flexible. If, in the bulk polymer, the secondary bonding, and modulus could be depressed, as described above, yet ultimate tensile properties maintained, a significantly improved prosthetic material could be realized.

* LD2699, now designated Adiprene LW520, E. I. Dupont, Wilmington, De. Lot numbers 107, 122, 131 .

$\dagger$ Aldrich Chemical Co., Milwaukee, Wi, Lot number $051217,99+\%$.

\$ Celanese Chemical Company, New York, NY.

§ Catalyst T-9, M\&T Chemicals, Inc., Rahway, NJ. Xenia, Orn.

* Drierite, W. A. Hammond Drierite Company,

if Linde 5A molecular sieve, 1/6-inch pellets.
The aim of this study was to formulate, prepare, and optimize the tensile properties of such a polymer, through control of the crosslink density and $\mathrm{NCO} / \mathrm{OH}$ ratio.

\section{Materials and Methods}

Poly(tetramethylene oxide) glycol, PTM$\mathrm{EG}$, and hexamethylene diisocyanate, HMDI, were selected as the macroglycol and diisocyanate respectively. These components were received in the prepolymer form* having a molecular weight of approximately $1,000 \mathrm{gm} /$ mole, and contain $4.75 \pm 0.15 \%$ active NCO end groups. A prepolymer system was selected because it can be cast into open molds, a preferred technique in producing maxillofacial prostheses. The PTMEG/HMDI pre-polymer was light amber in color and was a viscous liquid at room temperature. The pre-polymer was cured with the difunctional polyol 1,4butanediol, $\dagger$ and trifunctional trimethylol propane, TMP. Because of the low reaction rate between the aliphatic diisocyanate and polyol curing agent, a stannous octoate catalyst, $\S$ was incorporated at the time of mixing.

The prepolymer was received sealed under nitrogen. After each use, the metal container was purged with dry nitrogen and resealed. This procedure was also used with the curing agents and catalyst, which were stored in a desiccator between uses. The nitrogen was dried by bubbling it through sulfuric acid, passing it through a column of sodium hydroxide pellets, and finally through a column of anhydrous calcium sulfate.* It was critical that moisture contamination be avoided because of the hydrophilic nature of the reactants. Contaminated materials could upset the stoichiometric balance of the polyurethane reaction.

The prepolymer casting technique was developed from the supplier's recommendations as well as suggestions from other researchers, ${ }^{20}$ and consisted of the following steps:

1. The desired ratios of 1,4-butanediol and trimethylolpropane were mixed. TMP is a solid at room temperature, but if both polyols are heated and mixed, the resulting solution is liquid at room temperature, facilitating further handling.

2. Twenty four hours before sample preparation, the catalyst was accurately weighed into the curing agent mixture. Approximately one tablespoon of molecular sieve $\dagger$ was added per liter of catalyst-cure to remove any remain- 
ing moisture. The entire mixture was slowly rotated for 24 hours before sample preparation.

3 . The prepolymer was carefully weighed into a disposable polypropylene beaker and heated to $100 \mathrm{G}$ under a $5 \mathrm{~mm} \mathrm{Hg}$ vacuum in a 2,000-ml reaction kettle. Degassing was considered complete when excessive bubbling of the prepolymer stopped.

4. Simultaneously with step 3 , a stoichiometric excess of the cure-catalyst mixture was poured into a second disposable polypropylene beaker, heated and degassed in a reaction kettle.

5. When thoroughly degassed and heated to $100 \mathrm{C}$, the prepolymer was removed from the reaction kettle and reweighed. Using an eyedropper, the cure-catalyst mixture was carefully weighed into the prepolymer. The curecatalyst mixture was added to within $1 \%$ of the desired amount. This step was accomplished in 60 seconds.

6 . The prepolymer-cure-catalyst mixture was mixed for 60 seconds with a variable speed stainless steel propeller. Mixing speed was controlled to allow maximum mixing without whipping bubbles into the liquid polymer.

7. The liquid polymer was replaced into the reaction kettle and degassed for an additional 120 seconds at $100 \mathrm{C}$ and $5 \mathrm{~mm} \mathrm{Hg}$.

8. The liquid polymer was then cast into preheated, fluorocarbon coated, ${ }^{*}$ stainless steel molds. Tensile specimens for the polyetheraliphatic prepolymer were compression-molded and cured in the press at $100 \mathrm{C}$ for 8 hours.

The optimum stoichiometry of the aliphatic-polyether elastomer for maxillofacial application was determined by simultaneously varying the crosslink density and prepolymer to curing agent ratio. The crosslink density was controlled by reacting the prepolymer with various 1,4-butanediol/TMP ratios. Increased amounts of TMP resulted in higher crosslinked densities. The various ratios of 1,4butanediol/TMP along with the resulting molecular weights per crosslink, Mc, are listed in Table 1. The total equivalent weight of curing agent to prepolymer, identified as the $\mathrm{OH} /$ NCO ratio, was varied from a $10 \%$ deficiency, to stoichiometric amount, to $10 \%$ excess.

Five dumbbell-shaped tensile specimens were prepared with each different 1,4-butanediol/TMP and OH/NCO combination, for a total of 105 specimens. Each group of five

\footnotetext{
* Fluoro Glide, Chempast, Inc., Wayne, NJ.

$\doteqdot$ Instron Corporation, Canton, Ma.
}

TABLE 1

\section{1,4-Butanediol/Trimethylolpropane Ratios and Resulting Molecular Weights PER GROSSLINK}

\begin{tabular}{cc}
\hline $\begin{array}{c}\text { 1,4-Butanediol/TMP } \\
\text { Ratio }\end{array}$ & $\begin{array}{c}\text { Molecular Weight } \\
\text { Per Crosslink, Mc }\end{array}$ \\
\hline $0 / 100$ & 2,600 \\
$25 / 75$ & 3,400 \\
$50 / 50$ & 5,100 \\
$60 / 40$ & 6,400 \\
$70 / 30$ & 8,600 \\
$80 / 20$ & 12,900 \\
$100 / 0$ & No primary crosslinking \\
\hline
\end{tabular}

specimens was poured from one batch of material, and compression-formed in the same mold. Catalyst concentration was adjusted for each batch so that all specimens were molded 10 to 15 minutes after mixing of the prepolymer and curing agent. The fluorocarbon-coated stainless steel mold was recoated after every four to six uses. All samples were inspected at $5 \times$ magnification for nicks, tears, and bubbles. Defective samples were discarded. Molded tensile specimens had gauge dimensions of $3.2 \times$ $3.2 \times 25.4 \mathrm{~mm}(\mathrm{I} / 8 \times 1 / 8 \times 1 \mathrm{inch})$. All tensile samples were tested on a constant strain-rate testing machine, $\uparrow$ at room temperature, using a crosshead speed of $5 \mathrm{~cm} / \mathrm{min}$. Strain was measured with an incremental extensometer, $\dagger$ using a one-inch gauge length.

\section{Results}

Overall, there was a greater variation in properties with the 1,4-butanediol/TMP ratio than with the $\mathrm{OH} / \mathrm{NCO}$ ratio. This effect is understandable since the former ratio has a more drastic effect on crosslink density. With increasing relative amounts of the difunctional curing agent 1,4-butanediol, and consequently a decrease in crosslink density, there was a general increase in strength and a fourfold increase in elongation. The final elongation is plotted as a function of molecular weight per crosslink, $\mathrm{Mc}$, for the three different $\mathrm{OH} / \mathrm{NCO}$ ratios in Figure 2.

For any given crosslink density, there was a relatively small change in tensile properties with $\mathrm{OH} / \mathrm{NCO}$ ratio. This observation was particularly true under $250 \%$ elongation. Figure 3 contains the stress-elongation data for the samples prepared with a 70/30 1,4-butanediol/ TMP ratio, and is representative of the effect 


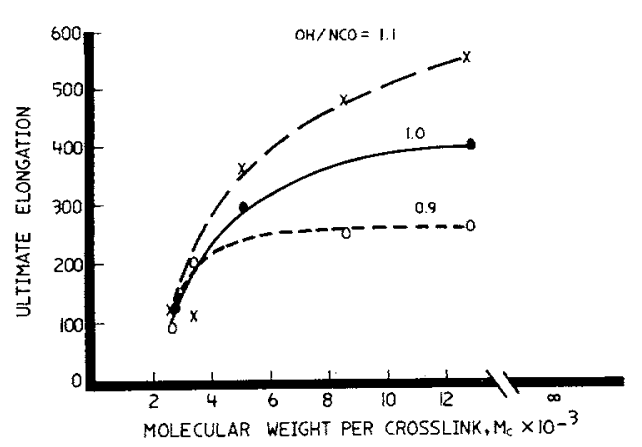

FIG 2.-_Ultimate elongation versus molecular weight per crosslink for the aliphatic/polyether system; $\mathrm{OH} / \mathrm{NCO}=0.9,1.0,1.1$.

of varying the $\mathrm{OH} / \mathrm{NCO}$ ratio with a given Mc. There was a slight depression of the $100 \%$ modulus (stress at 100\% elongation), but an increase in ultimate tensile strength and final elongation, with increasing $\mathrm{OH} / \mathrm{NCO}$ ratio.

The stress-elongation curves representing the various 1,4-butanediol/TMP ratios, but with a common $\mathrm{OH} / \mathrm{NCO}$ ratio of 1.0 are grouped in Figure 4. This figure, in essence, shows the change in tensile properties with crosslink density. The calculated molecular weight per crosslink is shown for each curve.

The stress-elongation curves measured at constant crosshead speed and temperature were typically nonlinear, and represented the time-dependent viscoelastic properties of these elastomers. For maxillofacial prosthetic application the modulus of the material is of particular importance, because flexibility affects biocompatibility as well as esthetics. In order to

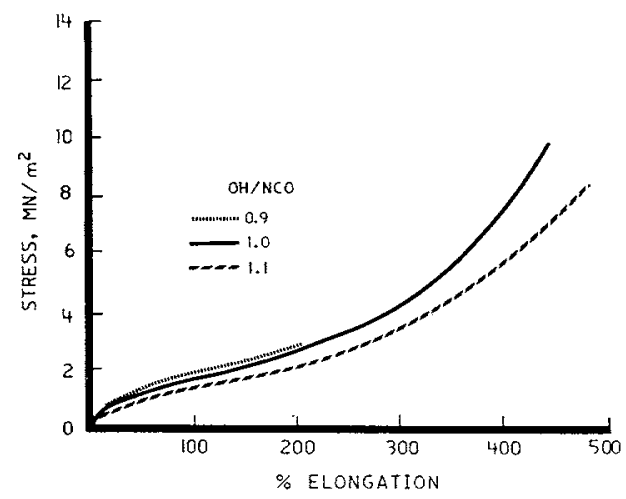

Fig 3.--Stress-elongation curves for the aliphatic/polyether system; 1,4-butanediol/ $\mathrm{TMP}=70 / 30, \mathrm{OH} / \mathrm{NCO}=0.9,1.0,1.1$. make a better comparison of the time-dependent stress-elongation data, the equilibrium values of the modulus were calculated following the scheme of Smith and Magnusson. ${ }^{30}$

The first step in calculating an equilibrium modulus is to establish a criteria which demonstrates that the values do in fact represent equilibrium behavior. The Smith and Magnusson treatment centers on the stressstrain equation predicted by the kinetic theory of elasticity rubber.

$$
\mathrm{S}=\operatorname{VRT}\left(\alpha-1 / \alpha^{2}\right)
$$

where $S$ is the engineering stress, $V$ is the moles of effective chains per units volume, $R$ is the gas constant, $\mathrm{T}$ is the absolute temperature, and $\alpha$ is the extension ratio equal to $\left[\Delta 1 / 1_{0}\right]+$ 1. These authors showed that a plot of $\alpha \mathrm{S}$ versus $\delta$, where $\delta$ equals $\Delta 1 / 1_{0}$, yields a straight line for most elastomers, and the slope yields a

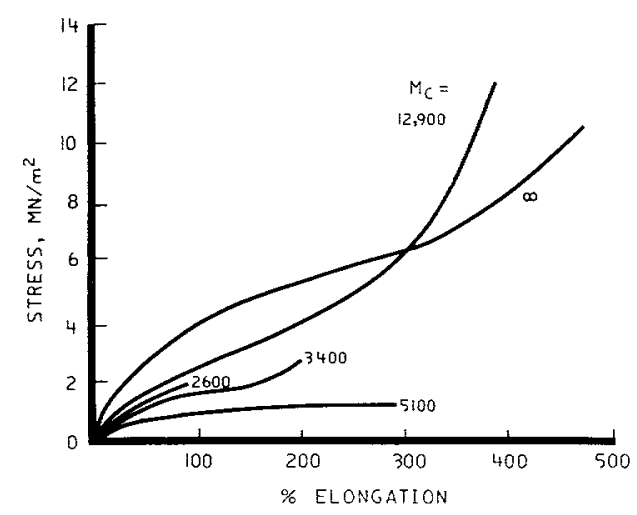

Fig 4.-Stress-elongation curves for the aliphatic/polyether system; OH/NCO $=1.0$.

precise value for the modulus, $\mathrm{E}$. The value of $\mathrm{E}$ thus calculated represents an equilibrium behavior if the slope of the $\log (\alpha \mathrm{S})$ versus $\log (\delta)$ plot is unity. This stems from the fact that the exponent, $n$, in the equation

$$
\alpha \mathrm{S}=\mathrm{E} \delta^{\mathrm{n}}
$$

is unity for an equilibrium elastic response. The calculated value of $\mathrm{E}$ should be close to $3 \mathrm{G}$, where $\mathrm{G}$ is the bulk modulus obtained from the slope of a $S$ versus $\alpha-1 / \alpha^{2}$ plot.

Using the stress-elongation data from Figure 4 , plots of $\alpha \mathrm{S}$ versus $\delta$ (shown in Fig 5), $S$ versus $\alpha-1 / \alpha^{2}$, and $\log (\alpha S)$ versus $\log (\delta)$ were made. The slopes of the $\log (\alpha S)$ versus $\log (8)$ plots were linear, indicating that an 


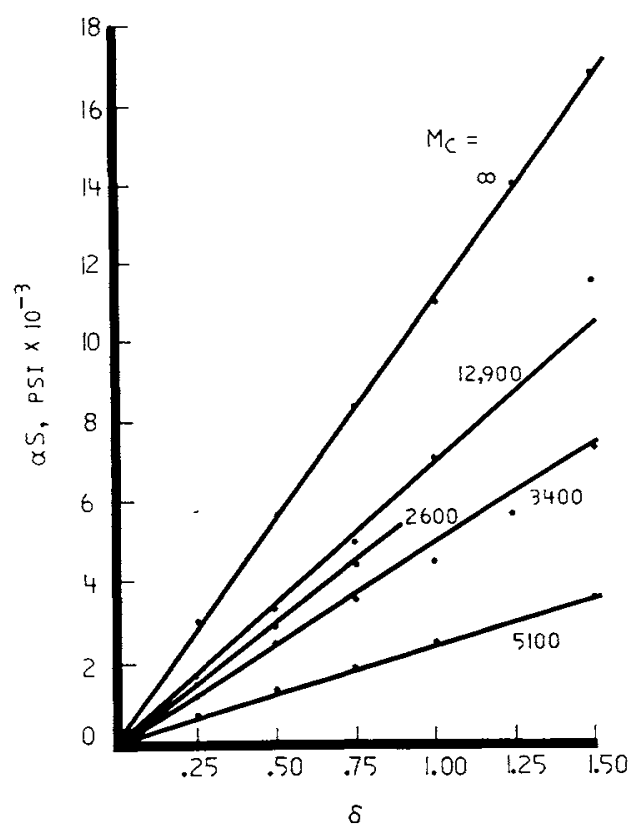

FIG 5.- S $\alpha$ versus $\delta$ for the aliphatic/polyether system calculated from the data in Figure 4.

equilibrium modulus was indeed determined. The modulus, $\mathrm{E}$, calculated from the $\alpha \mathrm{S}$ versus $\delta$ plots, the bulk modulus, $\mathrm{G}$, calculated from the $S$ versus $\alpha-1 / \alpha^{2}$ plots, and the slopes of $\log (\alpha \mathrm{S})$ versus $\log (\delta), \mathrm{n}$, are listed in Table 2.

\section{Discussion}

Although described in detail in the Methods section, several aspects of the technique used to polymerize the polyurethane elastomers warrant discussion. First, the importance of avoiding contamination by moisture cannot be understated. The presence of moisture during the reaction can completely eliminate the reactivity of the isocyanate groups on the urethane prepolymers. In the severe case, the polymerization can be minimal, resulting in a viscous liquid as a final product. Minor contamination allows polymerization, but the resultant molecular weight and crosslink density are below the theoretical values. Moisture contamination was minimized by maintaining all reactants and the catalyst in an atmosphere of dry nitrogen, and storing the containers in a desiccator. The use of the 5-Angstrom molecular sieve was an additional aid in removing moisture from the premixed curing agents. All handling and mixing was performed rapidly, and containers were purged and resealed as quickly as possible. Preparation of the urethane materials was suspended when conditions of high humidity existed in the laboratory.

Oxidation of the catalyst from the stannous to stannic octoate form is also a potential deterrent to achieving theoretical crosslink density and molecular weight. As indicated in the Introduction, the aliphatic urethane prepolymer required a catalyst for the curing reaction with 1,4-butanediol and trimethylol propane. It would have been desirable to cure the polyurethane without a catalyst, since this additive could potentially have a deleterious effect on the biocompatibility, and at higher temperatures, the hydrolytic stability of the final polymer. However, attempts at polymerization without a catalyst at higher tempera-

\section{TABLE 2}

Equilibrium Modulus, E, Bulk Modulus, G, Linearity Exponent, n, for the Poly (ether urethane) Elastomers, $O H / N C O=1$

\begin{tabular}{lcccc}
\hline \hline $\mathrm{Mc}$ & $\mathbf{n}$ & $\begin{array}{c}\mathrm{G}, \mathrm{MN} / \mathrm{m}^{2} \\
(\mathrm{psi})\end{array}$ & $\begin{array}{c}3 \mathrm{G}, \mathrm{MN} / \mathrm{m}^{2} \\
(\mathrm{psi})\end{array}$ & $\begin{array}{c}\mathrm{E}, \mathrm{MN} / \mathrm{m}^{2} \\
(\mathrm{psi})\end{array}$ \\
\hline 2,600 & 1.070 & 1.21 & 3.64 & 4.14 \\
& & $(176)$ & $(528)$ & $(600)$ \\
3,400 & 0.992 & 0.96 & 2.89 & 3.39 \\
& & $(140)$ & $(420)$ & $(492)$ \\
5,100 & 0.968 & 0.48 & 1.46 & 1.55 \\
& & $(70)$ & $(212)$ & $(225)$ \\
12.900 & 1.002 & 1.34 & 4.02 & 4.78 \\
& & $(194)$ & $(582)$ & $(693)$ \\
$\infty$ & 0.943 & 1.88 & 5.65 & 7.52 \\
& & $(273)$ & $(819)$ & $(1,090)$ \\
\hline
\end{tabular}


tures for even as long as 24 hours were unsuccessful. Stannous octoate was selected because it has been successfully used as a catalyst with siloxane polymers designed for biological environments. In the presence of moisture, the stannous octoate can be oxidized to a state which not only eliminates its ability to function as a catalyst, but actually inhibits the isocyanate-polyol reaction. This condition occurs if the relative amount of stannic octoate exceeds $8 \%$. Unfortunately, the change is not readily identified until all components have been mixed. The result is either no polymerization or a low molecular weight material, depending on the degree of contamination. Usually, the reaction can be accelerated with the incorporation of additional catalyst, although this approach is precarious at best and can lead to extremely fast reaction rates if excessive catalyst is used.

It is apparent then that incorporation of the curing agents and catalyst into the heated urethane prepolymer must be rapid to avoid moisture contamination, but also accurate. This procedure was accomplished by re-weighing the prepolymer after heating at $100 \mathrm{G}$ and just prior to addition of the cure-catalyst mixture. The weights were recorded to within 0.01 gram and the necessary amount of cure-catalyst was calculated on this basis. With batches of approximately $50 \mathrm{gm}$, calculations indicated that the molecular weight per crosslink was controlled to within a value of $50 \mathrm{gm} / \mathrm{mole} /$ crosslink. The success in controlling morphology is reflected in the consistent change in properties with Mc.

Table 2 lists the equilibrium modulus values for the poly(ether urethanes) prepared with $\mathrm{Mc}$ values of 2,600 to infinity. These data agree with those of Pigott ${ }^{28}$ in that initially there is a depression of the modulus, followed by an increase in the modulus with crosslinking density. The minimum modulus occurs near an $\mathrm{Mc}$ value of 5,000. As described earlier, the decrease in modulus is caused by the interference in intermolecular bonds by the trimethylolpropane curing agent. The exponent " $n$ " values in Table 2, which theoretically should be unity, indicate that equilibrium modulus values were in fact obtained.

The polyurethane elastomer based on the aliphatic diisocyanate HMDI, and a polyether macroglycol, PTMEG, was selected on the basis of its potential physical as well as mechanical properties. The inherent chemistry of the reactants would provide good environmental stability, but the main challenge was to optimize, in one polymer, the mechanical properties necessary for a successful maxillofacial material. Tensile data were the primary determinants in this optimization process.

The elongation data in Figure 2 suggested that an $\mathrm{OH} / \mathrm{NCO}$ ratio of 1.1 would be desirable, since the lower isocyanate concentration resulted in a polymer with higher elongation. The ratio of 1.0 would have been acceptable, while the formations with $\mathrm{OH} / \mathrm{NCO}$ ratios of $0.9 \mathrm{had}$ insufficient elongation. The materials with $\mathrm{OH} / \mathrm{NCO}$ ratios of 1.1 had lower strengths, but not significantly below those samples with $\mathrm{OH} / \mathrm{NCO}$ ratios of 1.0 . Therefore, 1.1 was selected as the preferred $\mathrm{OH} /$ NCO ratio.

The crosslink density for the poly(ether urethane) was selected by reviewing the elongation data in Figure 2, the stress-elongation curves in Figure 4, and the equilibrium data in Figure 5. A minimum molecular weight per crosslink of approximately 6,000 was necessary for adequate elongation and strength. Mc values exceeding 13,000, however, resulted in higher than desirable values of modulus. Therefore, final formulations were selected which resulted in $\mathrm{OH} / \mathrm{NCO}$ ratios of 1.1 and molecular weights per crosslink between 6,000 and 13,000 . Two specific polymers were prepared for further testing. These polymers were based on 1,4-butanediol/trimethylol propane ratios of $80 / 20$ and $70 / 30$, which resulted in elastomers with $\mathrm{Mc}$ values of 12,900 and 8,600, respectively. Results of additional testing will be reported in subsequent articles.

\section{Conclusions}

A polyurethane elastomer based on an aliphatic diisocyanate and a polyether macroglycol can provide the necessary tensile properties for a satisfactory maxillofacial prosthetic material. An optimum balance of tensile properties for the poly(ether urethane) was obtained with a molecular weight per crosslink between 8,600 and $12,900 \mathrm{gm} / \mathrm{mole}$ and a $10 \%$ stoichiometric excess of the curing agents. The resulting polymer had a tensile strength of 11 $\mathrm{MN} / \mathrm{m}^{2}, 100 \%$ modulus of $2 \mathrm{MN} / \mathrm{m}^{2}$, and ultimate elongation of $500 \%$. Satisfactory properties were obtained for the poly(ether urethane) without the use of plasticizers or stabilizers. 


\section{References}

1. Bulbulian, A.H.: Facial Prosthetics, Springfield: Charles C Thomas, 1973.

2. CAstleberry, D.J.: Materials for External Prostheses; The Choices and the Challenge, Symposium on Dental Biomaterials-Research Priorities, NIDR, Des Plaines, Il, Aug 1973.

3. Firtel.i, D.N., and Bartlett, S.O.: Maxillofacial Prosthetics: Reproducible Fabrication, J Prosth Dent, 22:247-252, 1969.

4. Lepley, J.B., and Strauss, P.W.: Functional and Cosmetic Restoration of a Massive Orofacial Defect, J Prosth Dent, 30:635636, 1973.

5. Chalian, V.A.; Drane, J.B.; and StanDISH, S.M.: Maxillofacial Prosthetics: Multidisciplinary Practice, Baltimore: Williams and Wilkins Co., 1971, p 96.

6. LEWTS, D.H.; MiLler, D.R; and Cowsar, D.R.: Development of Improved Materials for Extraoral Maxillofacial Prostheses, P.H.S., N.I.D.R. Contract NOL-DE-42435, June 1976.

7. Robeson, L.M.; Saunders, W.J.; Chow, S.; and Matzner, M.: Development of Improved Materials for Extraoral Prostheses, P.H.S., N.I.D.R. Contract NOL-DE-42436, November 1976.

8. Lymann, D.J.; Kwan-Gett, C.; Zwart, H.H.J.; Bland, A.; Eastwood, N.; Kawat, J.; and KOLFF, W.J.: The Development and Implantation of a Polyurethane Hemispherical Artificial Heart, Trans Am Soc Artif Intern Organs, 17:456-463, 1971.

9. KolfF, T.; Burketr, G.; and Feyen, J.: Copoly (ether-urethanes) as Materials for Artificial Hearts, Biomater Med Devices Artif Organs 1:669-679, 1973.

10. Gardner, D.L.: Stability of Heparin Sulfur-35 Attached to a Modified Polyurethane Vascular Prosthetic, Trans Am Soc Artif Intern Organs, 15:7-14, 1969.

11. Brash, J.L.; Fritzinger, B.K.; and Bruck, S.D.: Development of Block CopolyetherUrethane Intraaortic Balloons and Other Medical Devices, $J$ Biomed Mater Res, 7: 313-334, 1973.

12. Boretos, J.W., and Pierce, W.S.: Segmented Polyurethane: A Polyether Polymer, J Biomed Mater Res 2:121-130, 1968.

13. Boretos, J.W.; Detmer, D.E.; and DonAGHy, J.H.: Segmented Polyurethane: A Polyether Polymer, II: Two Years Experience, J Biomed Mater Res, 5:373-387, 1971.

14. SKorNIK, W.A.; Dressier, D.P.; and RkiCARD, J.K.: Adherence of Prosthetic Skin, J Biomed Mater Res, 2:447-456, 1968.

15. Dressler, D.P.; BARLYN, L.; and SKorNTK, W.A.: Viable Prosthetic Interface, J Biomed Mater Res Symposium, 1:169-178, 1971.
16. Sharp, W.V.; Taylor, B.C.; Wright, J.; and FINELII, A.F.: Experience with Negatively Charged Polyurethane Backed Velours, I Biomed Mater Res Symposium, 1: 75-81, 1971.

17. Sharp, W.V.; Gardner, D.L.; and AndreSEN, G.J.: Bioelectric Polyurethane and Its Use in Internal Prostheses, U.S. Patent 3, 512,183 (cl. 3-1; a61f), 19 May, 1970.

18. Llewellyn-Thomas, E.; Wang, P.Y.; and Cannon, J.S.: Adhesion of Synthetic Organic Polymer on Soft Tissue. I. Fast Setting Polyurethane Adhesive, J Biomed Mater Res, 8:35-43, 1974.

19. Bernhard, W.F.; Cummin, A.S.; Harris, P.D.; and Kent, E.W.: New Flexible Vascular Adhesive for Use in Cardiovascular Surgery, Circulation, 27:739-741, 1963.

20. Sherman, R.T.: Evaluation of Polymers as Surgical Prostheses, U.S. Clearinghouse Fed. Sci. Tech. Inform., AD 1969, AD690700.

21. Phildips, R.W.: Science of Dental Materials, 7 th ed., Philadelphia: W. B. Saunders, $1973, \mathrm{p} 239$.

22. Saunders, J.H., and Frisch, K.C.: Polyurethanes, Chemistry and Technology, Technology, Parts $I$ and $I I$, in High Polymers $V$. $X V I$, New York: Interscience, 1962 and 1964.

23. Wright, P., and Cumming, A.P.C.: Solid Polyurethane Elastomers, New York: Gordon and Breach, 1969.

24. SAUnders, J.H.: Polyurethane Elastomers, in Polymer Chemistry of Synthetic Elastomer, Kennedy, J.P. and Tornquist, E.G.M., ed., New York: John Wiley, 1969.

25. FrISGH, K.C.: Recent Advances in the Chemistry of Polyurethanes, Rubber Chem Technol, 45:1442-1466, 1972.

26. Bonart, R.; Mobitzer, L.; and Hentze, G.: X-ray Investigations Concerning the Physical Structure of Crosslinking Urethane Elastomers. II. Butanediol as Chain Extender, J Macromol Sci Phys, 3:339-358, 1969.

27. Bonart, R.; Morbitzer, L.; and Mueller, E.H.: X-ray Investigations Concerning the Physical Structure of Grosslinking in Urethane Elastomers, III. Common Structure Principles for Extensions with Aliphatic Diamines and Diols, J Macromol Sci Phys, 9: 447-461, 1974.

28. Pigott, K.A., and Frye, B.F.: Data, $I$ Chem Eng, 5:391, 1960.

29. Dieter, C.: Personal Communication, Dept. of Polymer Science, University of Detroit, Detroit, Michigan, 1972.

30. Smith, T.L., and Magnusson, A.B.: Diisocyanate-Linked Polymers. II. Mechanical and Swelling Properties of Some Polyurethane Elastomers, $J$ Polym Sci, 42:391-416, 1960 . 